\title{
Wireless multimedia sensor network for rape disease detections
}

\author{
Zhou Libo ${ }^{1,2}$, Huang Tian ${ }^{2,3}$ and Guan Chunyun ${ }^{1 *}$
}

\begin{abstract}
In order to reduce pesticides, it is also necessary to understand how plant diseases originate in the beginning and to be around them all around the world. One of the best ways to achieve this goal is to promote images that are not visible to the world (WMSN) in an agricultural country. However, the sending of a wireless device with little knowledge of the images will add more traffic to traffic, especially electricity. In this pamphlet, we create a diagnostic procedure that is aimed at driving WMSN's additional resources. From the study of the locally available nodes of the plants, this new technique can make the first choice on the vegetation of the plants, knowing how to send pictures to the control center to continue to explore, to move forward. Look at the Internet. The same method includes the distribution of images using color and shape, and its 2D profile is used as part of the stages. What happens on metal images and lack of food indicates that the correct route is $94.5 \%$.
\end{abstract}

Keywords: Plant diseases, Object detection, Convolution neural networks, Filter banks, Rape diseases

\section{Introduction}

Agriculture is now changing from modern farming to modern agriculture. Agricultural marketing (IOT) will play a major role in promoting agricultural farming, including agricultural infrastructure development, the development of modern agricultural technology and agricultural use. The use of modern technology, computer technology, and computers is a computersimultaneous work of coordinating the development of smart farming and productive farming [1].

Crop rotation as a major activity for agricultural material is of great importance [2]. Because of climate change, it is not only necessary to do a thorough investigation and research on the growth of the crop, as well as to understand the small crop yields. The growth of the crop and the growth of crop yields provides scientific and agricultural support. In order to maximize the profitability of farming, various species of biodiversity and biodiversity were established. The crop rotation system also confirms two types of information and reengineering. The room for environmental creation produces heat and soil, such as heat, humidity, wind, wind,

\footnotetext{
* Correspondence: guancy188@163.com

${ }^{1}$ College of Agriculture, Hunan Agricultural University, Changsha 410128, China

Full list of author information is available at the end of the article
}

rain, $\mathrm{pH}$ prices and so on [3]. Panel drawing photo gets a picture of the size of the plant. The size and size of the crop can be shown directly. Many numbers make up a moth-free area, and they can use the Internet.

The whole story is being prepared in this way. The second phase creates the design of the hardware platform. The third stage, the provision of scalar sensor node is asked. Then in the fourth stage, the formation of a photo snapshot picture is described. The fifth stage, the program is launched. The sixth stage produces test results. Finally, summarize this pamphlet.

\subsection{Related works}

Work offices use WSN node (or node) to learn more about the soil and send data to archives. Micro devices have sensors, systems and communication trips. The key points to consider are the need to use data, time and resources for communication, the size of the connection to node and sensors, crop requirements and so on. These articles are discussed in this section. Because the sequence of the list is divided into size groups, each part of the field is managed by one or more groups made up of one or more WSN additions. Soils will have more information about soil, climate, and / or crops. When airplane travels at each camp, the data is sent to a storage 
facility, which sends packages through the UAV airplane, which is represented as an airplane.

This natural body has been described as a comprehensive web site $[4,5]$. It also allows for node sections to be explained in accordance with minimum requirements and requirements, regardless of communication. In this mode, the number and functions of node are upping as these factors are used to establish and grow in each field. In this sense, the area where the height of height is not too high (eg, temperature) will be monitored by a number. Instead, neighboring glasses should be used to monitor changes in amplitude (eg gas, shaking). Good bulletin and internet system are proven by trials: from the christmas movement, the number of natural tendencies is learned to describe big changes. With these data, the particles can be moved quickly, and the example used in the inner location is found [6].

However, communication skills should also take into account. The delivery of windows should re-do dust work, but to try to create a mesh bullet that permits full cooperation between the nodes nearby. It allows all the data to be stored by placing the flight number in the jungle, so avoid the need to find it correctly. Access to more information from any neighboring location also contributes to the best way to solve each phase. It seems to make the whole world run smoothly and causes problems related to communication [7]. This process works best if there is no bad grid that is meant to be originally meant for the environment, but without working properly. When node distribution really causes the spread of stories about communication becomes important only. Even at this time, construction can make sure that the work is more productive and increase the amount of time required. In addition, it is not only the way to prepare eggs for the eggs. As shown in these books, many of the methods used are described and described in a series of times that are sometimes outdated and timeconsuming, not working and waiting for regular communication [8]. However, the house will be full of overflowing sequence where the main package is being sent out from each point to reduce the harvest time [9].

Convolutional neural networks are now considered to be a great way to explore something. With advanced technology, the most efficient machines are the same. Among them, we mentioned the best known methods and notes. We settled on three recent construction projects: high-speed construction in the areas (Fast R-CNN) [10] in the paper, Single shot multi-box detector (SSD) [11] and network-based convolution network (R- FCN) [12]. As explained in [13], although the meta-column was originally planned by another device (VGG, ResNet etc.), we now use unequal enhancements for infrastructure. Therefore, all construction must be connected with any drawings, depending on the work or needs.
Fast R-CNN, the test process is executed out in two steps. Firstly, the manufacturing area (RPN) takes the picture as a reflection and preparation by drawing [11]. Medium tools are used to define conflicts; everyone has a score. In order to teach RPN, the plan to consider whether it includes the ironic idea of the object in terms of the IOU, between what they want and world standards. In the second section, a box that is already made is designed to lift objects from the same graph. Therefore, these damage are fed into additional iron sheets to showcase the potential of the modern listings and boxes in all areas. All of this is done through a collaborative partnership, allowing the system to share all the tools related to the change and form of interface, so that it can achieve unique limits. Since the start of the R-CNN start-up, it has been linked to a number of jobs due to its good performance in known knowledge by the district.

SSD infrastructure [14] uses feedforward network convolution to solve problems. Websites make up a regular group of boxes and availability of items in each box. The nets can do different things by combining prophecies from different groups with different answers. In addition to that, the SSD includes the process to become a single internet, thus avoiding the process of disruption process as well as timing time [15].

The R-FCN program [16] provides the use of graphs that can use to solve the problem of transformation. This proposal is analogous to a Fast $\mathrm{R}-\mathrm{CNN}$, but not from the same previews that are invading the region, but from the last areas that are shaking sideways (a region with greater chance of including things or being part of it) before it can [17]. By using this method, the storage capacity used in the local population is reduced. In [16], they demonstrated that using the ResNet-101 if a transparent device can make competitions compared to the Faster R-CNN.

So as to combat the type of wireless weaknesses such as quick communication, power consumption, limited use and power conservation and low-density, a mixed product consisted by three different sections is made: 1 . The search system. They are consisted from a machine with no electricity, which can manage the seed to make it known (for example, a good sample, storage, and drive). This is explained in detail in Section 2.1. 2. Mobile phone guide. The revised version is associated with the phone number that carries the vehicle and is used as the data collection section, described in section 2.2. 3 . Long-term communication Finally, a computer-based network service provider that offers long-term services. This is explained in section 2.3. The activity on the package is displayed on Fig. 1.

In order to meet the demands of the project, for example, to look at the distance between the garden and 

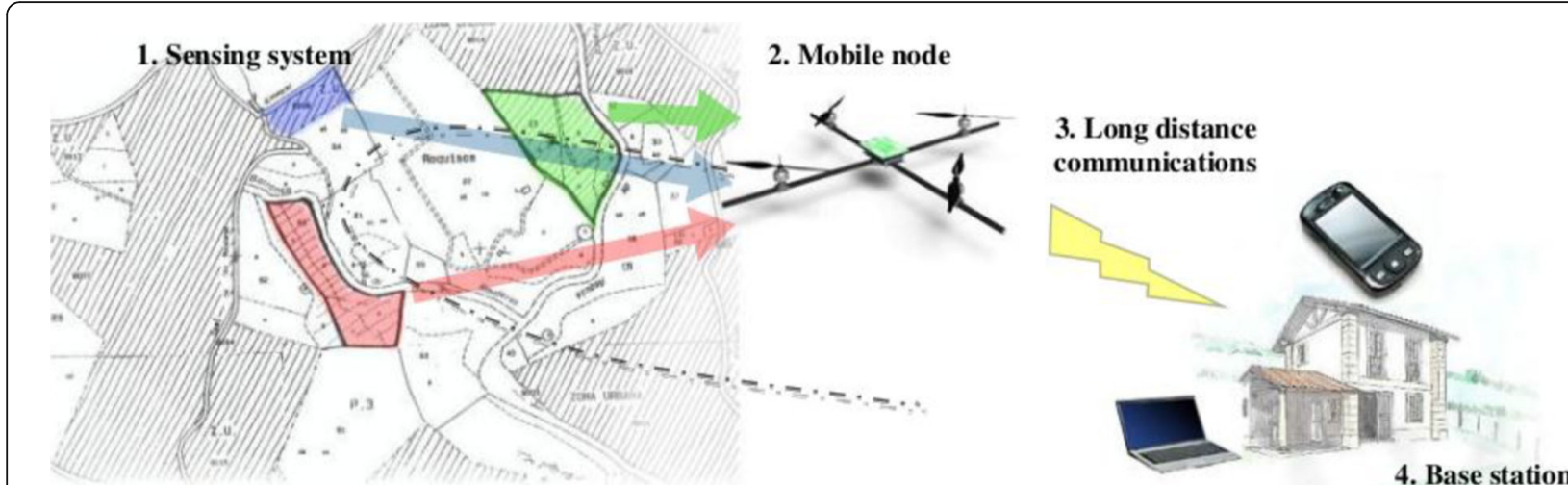

Fig. 1 An illustrative example of the overall remote sensing mission. The system layout on the package is shown in Fig. 1

the reserve, the package used for GPRS is allowed. Communication with an UAV database. GPRS connections allow data data to transmit from the dust of the vineyard until the train passes longer. The solution to this problem is just a few (QoS) of the catalyst machine, so it may seem like powerful and reliable. Unlike GPRS modules, modems placed on the UAVs allow data to be supplied to undermine the most advanced GPRS-outdoor areas outside of remote villages. The data is stored and then published as the UA arrives on the site with the interview. Another program is to use SMS (SMS), which allows regular messages to be sent to farm managers. To increase their knowledge. The above-mentioned method is based on the Arduino Board board and the GPRS team, which includes HiLo SAGEM method (see fig. 2).

This node is a self-representative SoC THLK2405 and CMOS photo drawing OV7640. Photo preview is included in SoC THLK2405. The graphic section includes three stages: the image area of the image, data capture and stress area. OV7640 offers additional display or image enhancements 8 in different words; is monitored by the web camera interface. OV7640 has images that can run faster for 30 frames per second, and run the image on the character of the photography, publishing

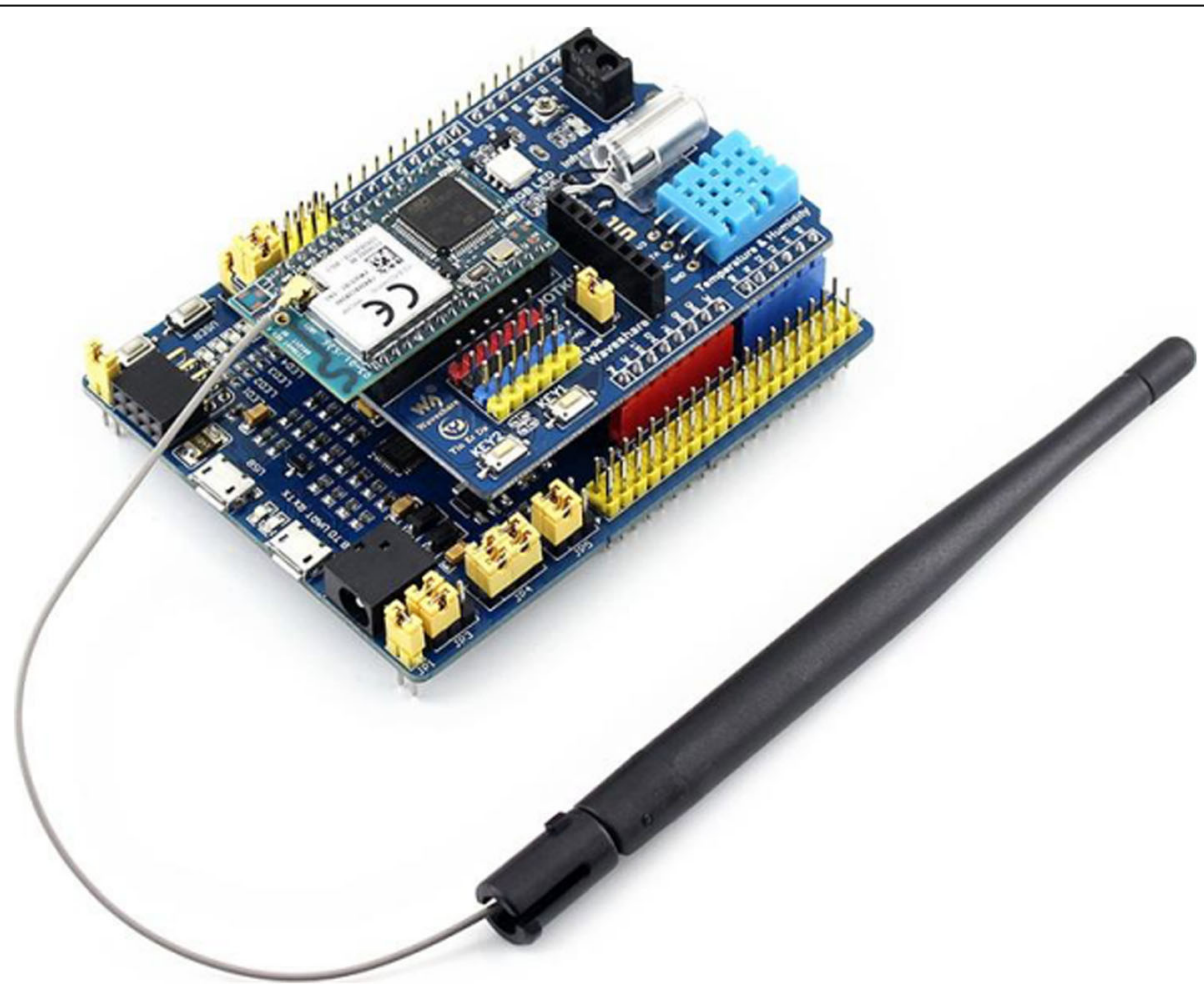

Fig. 2 Arduino system employed for communication with the base station, likewise for message delivery. The system mentioned above is based on the Arduino Uno board and the four-band GPRS module, which includes a HiLo SAGEM communication module (see Fig. 2) 
and publishing data. All the basic artwork, including ensuring ensuring, gamma, white cleanliness, color display, loss and so on, can also be processed through SCCB form. Figure 3 shows the headings and images of pictures.

\section{Methods}

Tomato trees are protected against many diseases and damage that cause disease and pests. Several reasons may be due to the harvest: (I) abiotic complications due to nature, such as heat, humidity, nutrients, fertilizers, bright colors and plants; (2) pathogens from plants to plants such as whitefly, vegetable gardens, worms, and so on. (3) Many diseases, including bacteria, viruses, and fungi. The disease is a pest, as well as a plant that has a variety of shapes, such as shape, color, shape, etc. Therefore, because of similarities, these changes are difficult to distinguish, which makes their awareness difficult, recognition and preparation can prevent many of the damage. Based on the information we have discussed, we consider the following characteristics:

- infection: according to the life cycle of the disease, plants show different patterns and their infection status.

- Location of symptoms: disease affects not only the leaves, but also the other location of the plant, such as stems.

- Leaf pattern: symptoms of disease show a marked change in the front or back of the leaf.
- Type of fungus: determining the size of fungus can be a simple way to distinguish clearly certain diseases.

- Color and shape: according to the disease, the main plants body may be look like different size at different time of infection.

Figure 4 showed the characteristic of different conditions and varying diseases and pests identified in our method. A detailed research of the symptoms of each class and pest is described in [18].

At each step, the window will sliding to a new location, it will predict the current region proposal at the same time, so the size of the reg layer will be 4 times larger than the number of the windows, compute the positions number of $\mathrm{k}$ boxes. Based on the research results of others, we found that using a variety of different feet the feature map of degree is predicted, and better results will be obtained. Therefore, we choose to use the output of 6 convolutional layers as the prediction layer of our network, which are res3b3 [19], res5c/conv1-2 [20], res5c/conv2-2 [21], res5c. /conv3-2, pool6. There will be two branches after each layer, one branch is responsible for predicting candidate frames, and one convolutional layer prediction candidate frame can be directly followed by the feature map. The other branch is responsible for predicting which category the candidate box belongs to [22]. Of course, each The candidate box for a layer of prediction and the number of categories it belongs to are fixed. Therefore, we can get 6 sets of candidate boxes, then we only need to combine the 6 sets of

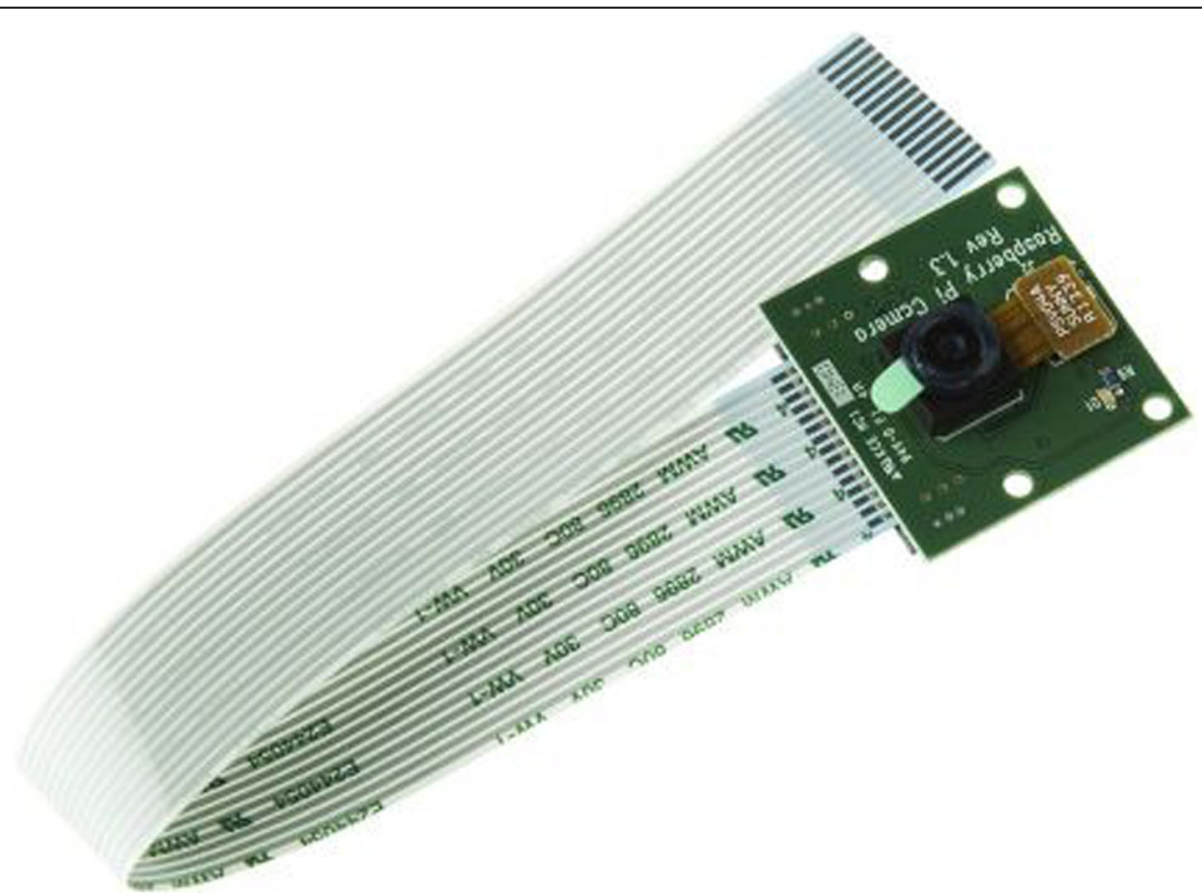

Fig. 3 The front and reverse sides of image sensor node. Figure 3 shows the heads and tails of the image nodes 

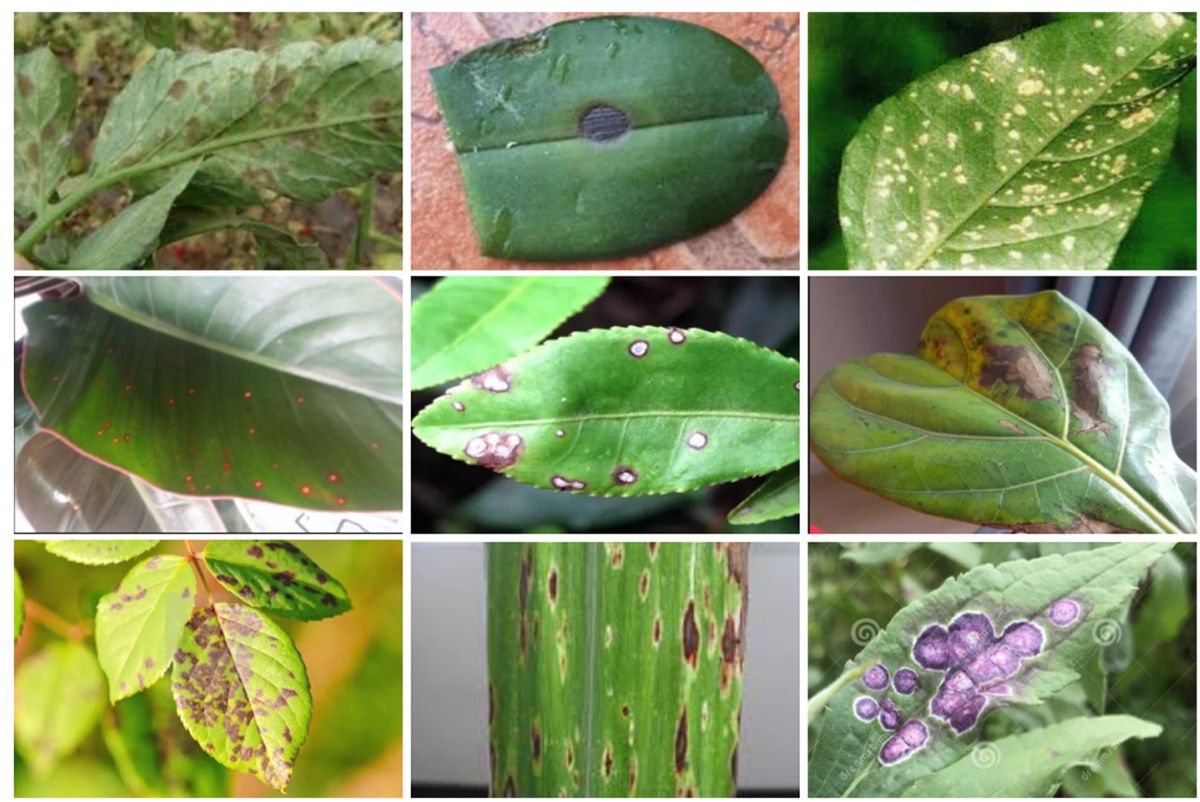

Fig. 4 The representative of diseases and pests affecting tomato plants. In Fig. 4, we show representatives of different conditions and varying diseases and pests identified in our work. (a) gray mold, (b) canker, (c) leaf mildew, (d) plague Yersinia pestis, (e) leaf miner, (f) whitefly, (g) low temperature, (h) nutrition excess or lack of, (i) powdery mildew. Collect images under different changing and environmental conditions. These patterns help to distinguish between certain appropriate characteristics of each disease and pest

candidate boxes together and calculate the loss function, then update the parameters of the network. The detailed structure of the network is described below.

For a complete target process, the first thing we need to do is to build a data set for our algorithm to learn. This data set should include the original image, as well as the location of each object in the original image. The detailed information we have Introduced in 2.2. Next we need to do a simple processing of the data set. Due to the algorithm used in this paper, we need to adjust the image to a fixed size. The fixed size used in this paper is $300 \times 300$. Next, we need to make an enhancement to the data, mainly because the training data included in this article is relatively small, so we need to increase the amount of data. Our loss function for the image is defined as:

$$
L=\frac{1}{N} \sum_{i} L_{c}\left(p_{i}, p_{i}^{\prime}\right)+\lambda \frac{1}{N} \sum_{i} p_{i}^{\prime} L_{r}\left(a_{i}, a_{i}^{\prime}\right)
$$

Here, $i$ is the index of a small number of anchors, and $p_{i}$ is the prediction probability of the anchor $i$ as an object. If the anchor is positive, the ground live label $p_{i}^{*}$ is 1 , and if the anchor is negative, it is $0 . t_{i}$ is the vector representing the four parameterized coordinates of the predictive boundary frame, and $t_{i}^{*}$ is the vector of the ground live frame associated with the positive anchor. Classification loss $L_{c l s}$ is the logarithmic loss of two classes (object and non-object).
For regression loss, we use $L_{\text {reg }}\left(t_{i}, t_{i}^{*}\right)=R\left(t_{i}-t_{i}^{*}\right)$, where $\mathrm{R}$ is a robust loss function (smooth L1) defined in [23].

For one of the feature maps [24] in each of the above layers, a solid number of candidate boxes. For example, if the size of the feature map is $m \times n$ and the number of candidate frames generated by each position is $\mathrm{k}$, then for a detection task of a class $\mathrm{c}$ object, $(\mathrm{c}+4) \times \mathrm{k} \times \mathrm{m} \times \mathrm{n}$ data. We call him the prior box. Here's how $\mathrm{k}$ is generated.

It is necessary to generate $\mathrm{k}$ differences in the same position of the feature map. The candidate box is because it can handle the problem of objects of different scales. The aspect ratio used in this paper is $\{2,3\}$. In the feature map of res3b3, we only use the aspect ratio of 2 , that is, in the layer of res3b3, each point on the feature map is generated. 3 candidate boxes, each of the other feature maps produces 4 candidate boxes. Thus we get $\mathrm{m} \times \mathrm{n} \times \mathrm{k}$ candidate boxes. The six candidate boxes consist of two squares, min_size $\times$ min_size, max_size $\times$ max_size, and the other two are calculated based on the aspect ratio and min_size. This way we have a lot of candidate boxes. Next we need to match the candidate box to the real box we have calibrated in our dataset to get the real box of our training. First we need to calculate the IOU [25] of the candidate box and the real box we generated in the current layer, and then select the positive sample with IOU greater than 0.5 , and the negative sample with less than 0.5. However, there is a big problem in doing 
this. Because we generate a candidate box at every position, there will be a large number of samples that are negative samples, so that we will have sample imbalance when training. The final training results have a relatively large impact. Therefore, we need to deal with the phenomenon of sample imbalance here, using focal loss to deal with such a situation, mainly to balance the negative and positive samples on loss. The method used in this paper is to use the difficult sample mining method. In this paper, the value of the loss function of each candidate box is calculated in the forward calculation, and then several candidate boxes with the largest loss function value are selected to be retained. Other candidates The box is directly discarded to ensure that the ratio of positive and negative samples in the training process is $3: 1$. In this way, we have obtained all the training data.

\section{Experiments/results}

We tried the difficulties in our Cole and pathogens, for example [17]. It includes about 5000 images collected from many Cole fields in Korea. Pathogens and diseases can be carried out in different areas such as weather, space and moisture. Thus, the images are collected in different ways according to time (such as lighting), weather (such as heat, humidity), and sweet surfaces (using a greenhouse). Additionally, our dataset includes photographs and variations, samples from the beginning, middle and end endings, images contained with different pages of plants (eg, stems, leaves, fruits, etc.), different plants, green make-up plants, etc. components Examples for each class are illustrated in Table 1. After the additional supplementary techniques, the number of annotation figures is similar to the set limits for the image in the image: geometric change (change, crop, fluctuation, fluctuation) and radical change (contrast to lighting, color, sound). The previous class is a horizontal class with a more detailed picture. When creating a $\mathrm{CNN}$ storage library, its box is used as an unpleasant example.

However, the alternate page view of Alternaria is a very similar type of rust, which determines their number. As shown in Fig. 5, an example of GoogLeNet Inception is used to remove the original statues of the image, making it more likely to deteriorate in different places. Therefore, the requirements used for $\mathrm{CNN}$ are a clear indication of the rape of the leaves.

In addition to the databases used [18], we have a new class with "yellow curl" images. As mentioned earlier, one of the major problems that we have found to reduce energy generated by the system is the balance between classrooms because of the limited resources and data available. This can be illustrated by the number of individual pictures, as shown in Table 1 and Fig. 6.

The size of the data cells affects the correctness of the apple's awareness. In this paper, two experimental teams are being conducted to show that the damage is real. The model was already taught and after data development. From the results shown in Fig. 7, it may be clear that the teaching method is a non-disclosure process, and eventually results in a total of $86.79 \%$. However, the additional requirements and policies make up $97.62 \%$ accurately, making this total by $10,83 \%$ compared to those that were not lost. It can be seen from the results which are known as, as shown in Fig. 7, this phenomenon is due to the following reasons: (1) Systematic arrangements that are made up of various specialized photography techniques provide many opportunities for learning how to apply models on CNN; (2) the spread of different types of photographs to the teaching of weight gain in the example from $\mathrm{CNN}$, but the photographic figure of the image is a wide range of variations and will cause serious problems; (3) The picture begins with the image of an apple photograph, so the CNN model can better appreciate the apple's character made from apple

Table 1 The list of the classes included in our Cole pest and disease dataset and its annotated samples

\begin{tabular}{cccc}
\hline Class & $\begin{array}{c}\text { Number of Images in } \\
\text { the Dataset }^{\mathbf{1}}\end{array}$ & $\begin{array}{c}\text { Number of Annotated } \\
\text { Samples (Bounding Boxes) }\end{array}$ & $\begin{array}{c}\text { Percentage of Bounding } \\
\text { Box Samples (\%) }\end{array}$ \\
\hline Leaf mold & 1350 & 11,922 & 27.47 \\
Gray mold & 335 & 2768 & 6.37 \\
Canker & 309 & 2648 & 6.10 \\
Plague & 296 & 2570 & 5.92 \\
Miner & 339 & 2946 & 6.78 \\
Low temperature & 55 & 477 & 1.09 \\
Powdery mildew & 40 & 338 & 0.77 \\
Whitefly & 49 & 404 & 0.93 \\
Nutritional excess & 50 & 426 & 0.98 \\
Background ${ }^{3}$ & 2177 & 18,899 & 43.54 \\
\hline Total & 5000 & 43,398 & 100 \\
\hline
\end{tabular}

${ }^{1}$ Number of images in the dataset; ${ }^{2}$ Number of annotated samples after data augmentation; ${ }^{3}$ Transversal category included in every image. 


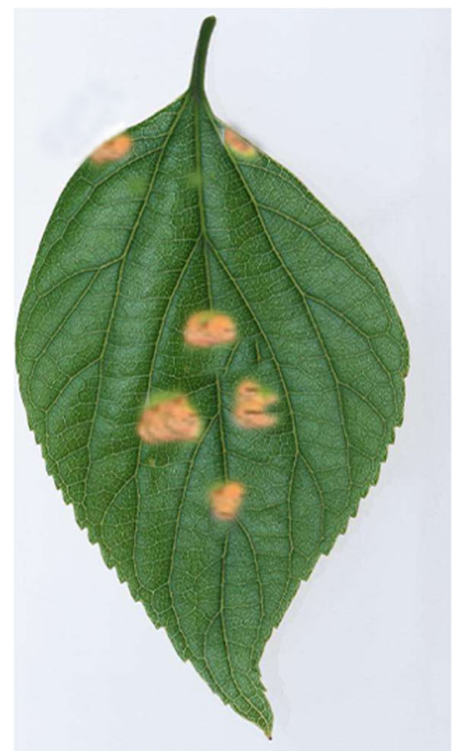

$\mathbf{a}$

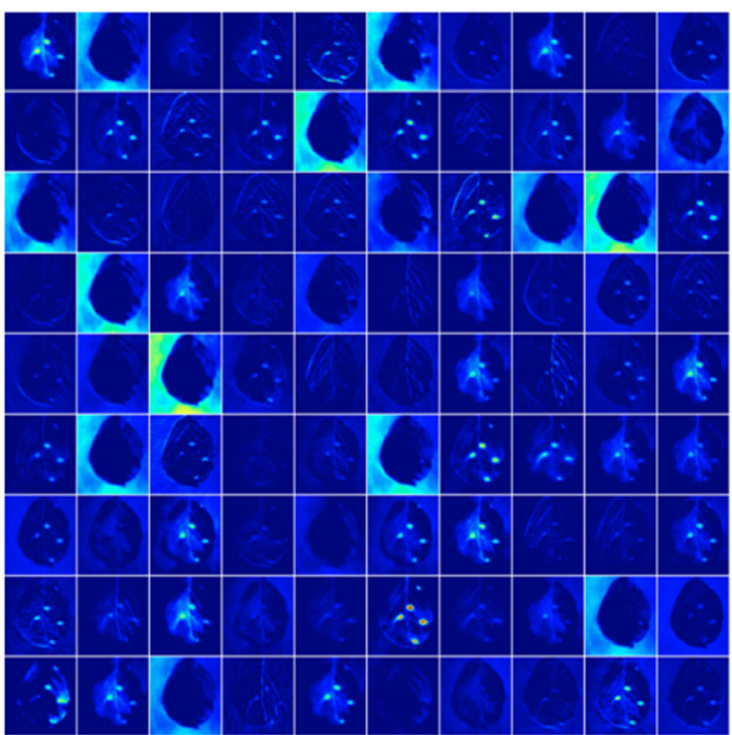

b

Fig. 5 Activation visualization: (a) original image; and (b) the learned weights by the first layer. As shown in Fig. 5, the model proposed by GoogLeNet Inception is used to extract the pathological features from the original image, which improves the automatic feature extraction in multidimensional space. Therefore, the proposed model based on CNN has better recognition ability for rape leaf disease

fruit. The display results show that data expansion may result in increased power levels.

Table 2 shows the final results of our refined system. The relative value proves that all categories have a satisfactory improvement compared to our previous results. The average accuracy has increased by about $13 \%$. In fact, this is due to the implementation of a secondary diagnostic unit (CNN filter bank) that enables the system to filter misclassified samples, with a primary focus on reducing false positives.

Sample size and change are another key point influencing the end result. For example, in the case of gray mold, the number of samples is less than that of leaf mold. In addition, the gray model class shows high intra-class variability, which may confuse the system with other classes (see Fig. 7).

\section{Discussion}

On this paper, a new way to get an UAV with wireless clocks (WSN) is run to look different from the plots. The following approach can overcome the problems associated with the distribution of interest-oriented areas, such as how long it is not possible to use WSN, related and continue to look at different issues. These problems have been supported by using a basic WSN mobile device that can always remember the subnetworks that are available in all regions. Because the movement organs are the ones that exist, it is easy to deal with problems related to soil conditions. We have been asking for a strong - known knowledge of mysterious learning known by pathogens. This process creates a cure for disease and the location of tomato trees, which represents a significant difference in other ways of fighting disease. Our
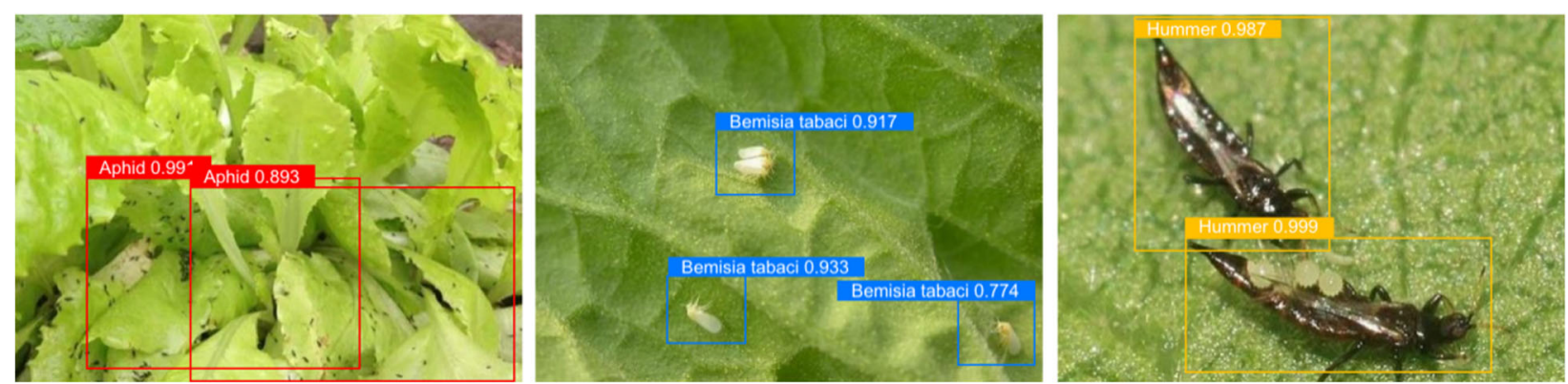

Fig. 6 Some False Positives Produced In The Primary Diagnostic Unit. Figure 6 shows some false positives produced in the primary diagnostic unit: (a) the ulcer sample was detected as plague; (b) the gray mold sample was detected as ulcer disease; (c) the low temperature sample was detected as ulcer disease 


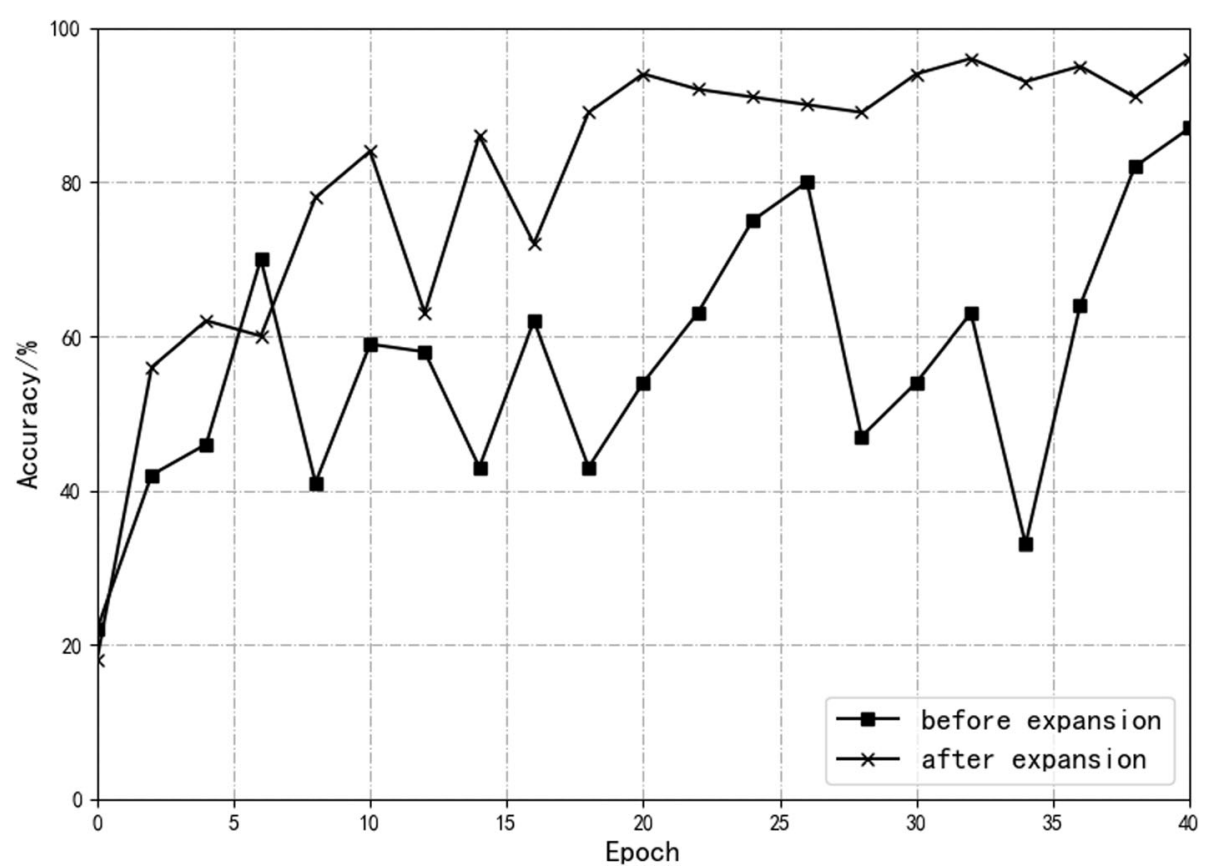

Fig. 7 The influence of expanded dataset. As shown in Fig. 7, this phenomenon is mainly due to the following reasons: (1) the extended datasets generated by various digital image processing techniques provide more opportunities for learning appropriate hierarchical features for models based on CNN; (2) the expansion of image diversity in image dataset is helpful to fully train the weight of learning in the model based on CNN, but the smaller image data set is lack of diversity and will lead to over-fitting problem; (3) the image preprocessing simulates the real collecting environment of apple pathological image, so the model based on CNN has better recognition ability to the natural apple pathological image obtained from apple orchard. The experimental results show that the extended data set can improve the generalization ability of the proposed model

review uses pictures that are carried out in situ with various camera tools and uses them using the latest technology and software programs using the GPU, rather than using a collection of natural resources (vegetation, vegetation) and research in the laboratory. In addition, our data on the diseases of tomato and insect pests have different functions, such as light, size, and so on. Our goal is to gain a deeper understanding of our work experience. Therefore, a comparison between the test results and the various depths of construction and the demonstration of how our detective detection system can find eight types of diseases and pests, including various and varied challenges. Additionally, we find that using advanced and more advanced technology can

Table 2 Comparison of our proposed method with other method

\begin{tabular}{cccccccc}
\hline \multicolumn{7}{c}{ Meta-Architectures } \\
\hline & \multicolumn{3}{c}{ Faster R-CNN } & & R-FCN & SSD \\
\hline $\begin{array}{c}\text { Class/Feature } \\
\text { Extractor }\end{array}$ & VGG-16 & ResNet-50 & ResNet-101 & ResNet-152 & ResNeXt-50 & ResNet-50 & ResNet-50 \\
\hline Leaf mold & $\mathbf{0 . 9 0 6 0}$ & 0.8827 & 0.803 & 0.8273 & 0.840 & $\mathbf{0 . 8 8 2 0}$ & 0.8510 \\
Gray mold & $\mathbf{0 . 7 9 6 8}$ & 0.6684 & 0.449 & 0.4499 & 0.620 & $\mathbf{0 . 7 9 6 0}$ & 0.7620 \\
Canker & $\mathbf{0 . 8 5 6 9}$ & 0.7580 & 0.660 & 0.7154 & 0.738 & $\mathbf{0 . 8 6 3 8}$ & 0.8326 \\
Plague & $\mathbf{0 . 8 7 6 2}$ & 0.7588 & 0.613 & 0.6809 & 0.742 & $\mathbf{0 . 8 7 3 2}$ & 0.8409 \\
Miner & 0.8046 & 0.7884 & 0.756 & 0.7793 & 0.767 & 0.8812 & 0.7963 \\
Low temperature & 0.7824 & 0.6733 & 0.468 & 0.5221 & 0.623 & 0.7545 & 0.7892 \\
Powdery mildew & 0.6556 & 0.5982 & 0.413 & 0.4928 & 0.505 & 0.7950 & 0.8014 \\
Whitefly & 0.8301 & 0.8125 & 0.637 & 0.7001 & 0.720 & 0.9492 & 0.8402 \\
Nutritional excess & 0.8971 & 0.7637 & 0.547 & 0.8109 & 0.814 & 0.9290 & 0.8553 \\
Background & 0.9005 & 0.8331 & 0.624 & 0.7049 & 0.745 & 0.8644 & 0.8841 \\
Total mean AP & $\mathbf{0 . 8 3 0 6}$ & 0.7537 & 0.590 & 0.6683 & 0.711 & $\mathbf{0 . 8 5 9 8}$ & 0.8253 \\
\hline
\end{tabular}

* The bold numbers correspond the more challenging classes and best results among other meta-architectures. AP: average precision. 
achieve better performance. We hope that our prepared program will play a key role in the research process. Future work will improve the future results and further work will spread the knowledge of disease and insects to other plants.

The main purpose of the project is to use the UAV as a link to airline and to fix WSN change when the UAV is near your target area. Ad-hoc communications and communication systems have been used to solve these problems. In addition, the establishment of a user interface, can deal with data and monitor data. We need to emphasize that the cheap and reliable UAV platforms are now available and can be easily used for such purposes. The project provides an opportunity to try using four automobile vehicles in real-life situations. The UAV can stop the project and fly over a different distance.

\section{Abbrevistions}

ANN: Artificial Neural Network; BP: Back-Propagation; CNN: Convolutional Neural Network; LDA: Linear Discriminant Analysis; MLP: Multi-Layer Perceptron

\section{Acknowledgements}

Not applicable.

\section{About the author's}

Libo Zhou received his bachelor's degree in Physics from Hunan University of Science and Technology in 2001 and his master's degree in software engineering from Hunan University in 2012. He is currently pursuing a doctorate at Hunan Agricultural University in Changsha, China. His main research interests include image processing, image recognition algorithms, and high-speed information collection and processing.

Huang Tian received the B.S degree in aeronautical power plant contro engineering from Nanjing University Of Aeronautics and Astronautics, Nanjing, China, in 1983, and the M.S. degrees in automatic control theory and application from National University of Defense Technology, Changsha, China, in 1988, and the Ph.D. degrees in mechanical manufacture and automation from Nanjing University Of Aeronautics and Astronautics, Nanjing, China, in 1992, he worked as a professor at the School of Information Science and Engineering of Central South University, Changsha, China. His research interests include artificial intelligence and application, bionic robot and intelligent bionic system, advanced control theory and advanced calculation, biomedical image processing.

Chunyun Guan Professor Guan, a mongolian ethnic, is an academician of Chinese Academy of Engineering, a leading doctoral mentor of the first-calss subject of crop science, and a doctoral and MPhil mentor of the subject of plant cultivation.

As a visiting senior scholar, he studied in Univesity of Alberta in Canada from 1993 to 1994. In the following five years (1995-2000), he was president of HUNAU. Currently, he is head of the Research Institute of Oil-bearing Crops of HUNAU; chief of the provincial key laboratory of crop genetic engineering in Hunan; and the person-in-charge of the Hunan Branch of the National Center for Improvement of Oil-bearing Crops. He also holds important posts such as: the councilman of Groupe Consultatif International de Recherche sur le Colza (GCIRC); vice-chairman of Hunan Scientific Institution; the convener of the Second Discipline Evaluation Subcommittee of the State Degree Committee; and an expert of the Discipline Evaluation Committee of the State Council.

Scope of Research: Modern Breeding of Rapeseeds and its Molecular Basis, ecophysiology and molecular biology of rapeseed, cultivation methods of high-yielding and quality rapeseed and utilization of such products.

\section{Availability of data and materials}

The datasets used and/or analyzed during the current study are available from the corresponding author on reasonable request.

\section{Authors' contributions}

LBZ designed the study, performed the experiments, and data analysis, and wrote the paper. LBZ and TH advised on the design of the system and analyzed to find the best method for efficient recognition of diseases and pests of Cole plants. GC provided the facilities for data collection and contributed with the information for the data annotation. All authors read and approved the final manuscript.

\section{Competing interests}

The authors declare that they have no competing interests.

\section{Publisher's Note}

Springer Nature remains neutral with regard to jurisdictional claims in published maps and institutional affiliations.

\section{Author details}

${ }^{1}$ College of Agriculture, Hunan Agricultural University, Changsha 410128, China. ${ }^{2}$ College of Information and Electronic Engineering, Hunan City University, YiYang 413002, Hunan Province, China. ${ }^{3}$ Hunan Engineering Research Center for Internet of Animals, Changsha 410205, China.

Received: 27 January 2019 Accepted: 3 May 2019

Published online: 14 June 2019

\section{References}

1. G. Owomugisha, E. Mwebaze, in Proceedings of the 2016 15th IEEE International Conference on Machine Learning and Applications (ICMLA), Anaheim, CA, USA, 18-20. Machine learning for plant disease incidence and severity measurements from leaf images (2016)

2. M. Dutot, L.M. Nelson, R.C. Tyson, Predicting the spread of postharvest disease in stored fruit, with application to apples[]]. Postharvest Biol. Technol. 85, 45-56 (2013)

3. P. Zhao, G. Liu, M.Z. Li, et al., Management information system for apple diseases and insect pests based on GIS[J]. Trans. Chin. Soc. Agric. Eng 22, 150-154 (2006)

4. Y. Es-saady, I. El Massi, M. El Yassa, et al., in 2016 International Conference on Electrical and Information Technologies (ICEIT). IEEE. Automatic recognition of plant leaves diseases based on serial combination of two SVM classifiers[C] (2016), pp. 561-566

5. P.B. Padol, A.A. Yadav, SVM classifier based grape leaf disease detection[C]// Advances in Signal Processing. IEEE, 175-179 (2016)

6. S.S. Sannakki, V.S. Rajpurohit, V.B. Nargund, et al., "Diagnosis and classification of grape leaf diseases using neural networks"[C]// 2013 Fourth International Conference on Computing, Communications and Networking Technologies (ICCCNT). IEEE, 1-5 (2013)

7. T. Dean, M.A. Ruzon, M. Segal, et al., in Proceedings of the IEEE Conference on Computer Vision and Pattern Recognition. Fast, accurate detection of 100,000 object classes on a single machine[C] (2013), pp. 1814-1821

8. J. Donahue, Y. Jia, O. Vinyals, et al., in International Conference on Machine Learning. Decaf: A deep convolutional activation feature for generic visual recognition[C] (2014), pp. 647-655

9. Zeiler M D, Fergus R. Stochastic Pooling for Regularization of Deep Convolutional Neural Networks[]]. arXiv preprint arXiv:1301.3557, 2013

10. J. Dong, Q. Chen, S. Yan, et al., in European Conference on Computer Vision. Towards unified object detection and semantic segmentation[C] (Springer, Cham, 2014), pp. 299-314

11. D. Erhan, C. Szegedy, A. Toshev, et al., in Proceedings of the IEEE Conference on Computer Vision and Pattern Recognition. Scalable object detection using deep neural networks[C] (2014), pp. 2147-2154

12. M. Everingham, S.M.A. Eslami, L. Van Gool, et al., The pascal visual object classes challenge: A retrospective[J]. Int. J. Comput. Vis. 111(1), 98-136 (2015)

13. P.F. Felzenszwalb, R.B. Girshick, D. McAllester, et al., Object detection with discriminatively trained part-based models[J]. IEEE Trans. Pattern Anal. Mach. Intell. 32(9), 1627-1645 (2010)

14. S. Gidaris, N. Komodakis, in Proceedings of the IEEE International Conference on Computer Vision. Object detection via a multi-region and semantic segmentation-aware cnn model[C] (2015), pp. 1134-1142

15. S. Ruder, An Overview of Gradient Descent Optimization Algorithms[J]. arXiv preprint arXiv:1609.04747 (2016) 
16. A. Krizhevsky, I. Sutskever, G.E. Hinton, in Advances in neural information processing systems. Imagenet classification with deep convolutional neural networks[C] (2012), pp. 1097-1105

17. C. Szegedy, W. Liu, Y. Jia, et al., in Proceedings of the IEEE Conference on Computer Vision and Pattern Recognition. Going deeper with convolutions [C] (2015), pp. 1-9

18. A. Giusti, D.C. Cireşan, J. Masci, et al., in 2013 IEEE International Conference on Image Processing. IEEE. Fast image scanning with deep max-pooling convolutional neural networks[C] (2013), pp. 4034-4038

19. P. Pawara, E. Okafor, O. Surinta, et al., Comparing Local Descriptors and Bags of Visual Words to Deep Convolutional Neural Networks for Plant Recognition[C]//ICPRAM (2017), pp. 479-486

20. M. Holmberg, F.A.M. Davide, C. Di Natale, et al., Drift counteraction in odour recognition applications: Lifelong calibration method[]]. Sensors Actuators B Chem. 42(3), 185-194 (1997)

21. Cugu I, Sener E, Erciyes C, et al. A Novel Tree Classifier Utilizing Deep and Hand-Crafted Representations. arXiv 2017. arXiv preprint arXiv:1701.08291

22. J. Amara, B. Bouaziz, A. A Deep Learning-based Approach for Banana Leaf Diseases Classification[C]//BTW (Workshops). 79-88 (2017)

23. Bahrampour S, Ramakrishnan N, Schott L, et al. Comparative Study of Caffe, Neon, Theano, and Torch for Deep Learning[J]. 2016

24. Y. Kawasaki, H. Uga, S. Kagiwada, H. Iyatomi, in Advances in Visual Computing, Proceedings of the 11th International Symposium, ISVC 2015, Las Vegas, NV, USA, 14-16 December 2015, ed. by G. Bebis. Basic Study of Automated Diagnosis of Viral Plant Diseases Using Convolutional Neural Networks, vol 9475 (Lecture Notes in Computer Science; Springer, Cham, Switzerland, 2015), pp. 638-645

25. A. Johannes, A. Picon, A. Alvarez-Gila, et al., Automatic plant disease diagnosis using mobile capture devices, applied on a wheat use case[J]. Comput. Electron. Agric. 138, 200-209 (2017)

\section{Submit your manuscript to a SpringerOpen ${ }^{\circ}$ journal and benefit from:}

- Convenient online submission

- Rigorous peer review

- Open access: articles freely available online

- High visibility within the field

- Retaining the copyright to your article

Submit your next manuscript at $\boldsymbol{\nabla}$ springeropen.com 